\title{
3D Position Detection with a PSD Using Frequency Modulated Light Markers
}

\author{
Kosuke Onishi, Daisuke Tanaka, Yuki Misaki, Shiyuan Yang \\ Kyusyu Institute of Technology
}

\begin{abstract}
The three-dimensional (3D) measurement method is used in various fields in recent years. However, the conventional 3D measurement method has various problems such as complicated pre-set, slow processing and high cost. In this study, in order to achieve the $3 \mathrm{D}$ measurement with high speed and low cost with easy preset than the conventional 3D measurement method, we propose the 3D measurement method with a two-dimensional Position Sensitive Device (2D PSD) using a pair of light sources.
\end{abstract}

Keywords: 3D measurement, 2D PSD, Pair light source.

\section{Introduction}

The 3D measurement method is used in various fields such as games, CG creation, structure research, cultural heritage, ruins investigation, robot vision and medical in recent years. In the future, the demand for the $3 \mathrm{D}$ measurement would increase with the development of $\mathrm{CG}$ technology.

The 3D measurement method can be largely divided into two types, the passive and active measurement. The passive measurement is the $3 \mathrm{D}$ measurement method using the camera only. The passive measurement is divided into the depth from focus method and the multi camera stereo method. The depth from focus method is measurement method using the different focal length for focusing depending on the object positions, and calculates the distance between the object and the camera based on focal length for giving best focusing. The multi camera stereo method is the $3 \mathrm{D}$ measurement method using multiple cameras, and calculates the 3D position based on the principle of triangulation. Because the depth from focus method is necessary to change the focal length by the sequential lens drive for distance measurement, the measurement of the moving object and real time measurement is difficult. Multi camera stereo method is an important and troublesome preset because the calibration determining the position relation of multiple cameras affects the accuracy. Also, the image processing of multi camera stereo method is time consuming. Furthermore, cost than other methods because it uses multiple cameras.

On the other hand, the active measurement is the $3 \mathrm{D}$ measurement method using the light, radio wave or sound wave as well as the camera. Active measurement is divided into light radar method and active stereo method. Light radar method is 3D measurement method using the time difference until the reflecting light reflected by the object is returned to the camera from irradiating. Active stereo method is the 3D measurement method using the information of the irradiating light consists of the camera and the unit for irradiating light to the object. Light radar method is not able to measure 3D when the object has an optical transmission and light absorption effects because this method utilizes the reflection of light. Active stereo method can determine the relative displacement of the object position but this method can't determine absolute distance. Also, active stereo method takes time for arithmetic processing because this method utilizes the pattern change of the light irradiated on the object.

In conventional 3D measurement, the 2D PSD had been used instead of the camera in the multi camera stereo method and the active stereo method. The 3D measurement using the camera requires one image to measure one point. Therefore, it was necessary to perform the 3D measurement $n^{2}$ times to scan the entire $n \times n$ [pixel] image. Therefore, rapid the 2D PSD in the response speed is used instead of the camera to accelerate series of processing of this method.

In this study, a pair of light sources is attached to the object. Furthermore, the 3D coordinate of the light source is calculated by using the $2 \mathrm{D}$ information from the 2D PSD. 


\section{Principle}

First, we project light of the light source attached to the target toward a 2D PSD module. This part we call the light projecting unit. Then, projected light is imaged by an optical lens on the 2D PSD light reception surface. This part we call the light receiving unit. The four output signals from the 2D PSD are A/D converted, and these data are calculated by the PC. Then, 2D coordinate of the spot light on the 2D PSD light reception surface is detected. 3D coordinate of the light source are detected by using 2D coordinate data. This part we call the calculation unit.

We show positional relationship between light source and 2D PSD in Fig. 1. Fig. 1(a) is the system schematic view, Fig. 1 (b) is the $X-Z$ plane view of the system, and Fig. 1 (c) is the $Y$ - $Z$ plane view of the system.

\subsection{Asynchronous measurement method}

Four output signals from the 2D PSD are the composite wave of different frequency sine waves of light sources. Four spectrums are obtained in the modulation frequency of light sources by Fast Fourier Transform (FFT). Four spectral peak voltages are $X 1, X 2, Y 1, Y 2$. Also, the length of the 2D PSD light reception surface is $c[\mathrm{~mm}]$.

$$
\begin{aligned}
& x=\frac{(X 2+Y 1)-(X 1+Y 2)}{X 1+X 2+Y 1+Y 2} \times \frac{c}{2} \\
& y=\frac{(X 2+Y 2)-(X 1+Y 1)}{X 1+X 2+Y 1+Y 2} \times \frac{c}{2}
\end{aligned}
$$

Therefore, the 2D coordinate of light sources on the 2D PSD light reception surface is detected simultaneously.

\subsection{D measurement method}

From Fig. 1 (b) and (c), the $\Delta x[\mathrm{~mm}]$ is the interval of a pair of light sources on the 2D PSD light reception surface. Also, the $\Delta X[\mathrm{~mm}]$ is the arbitrary interval of a pair of light sources. Furthermore, the distance $L[\mathrm{~mm}]$ is as follows based on the similarity relationship of triangle.

$$
L=\frac{\Delta X}{\Delta x} d
$$

From Fig. 1 (b) and (c), the 3D coordinate of each light source $(X, Y, Z)$ is as follows. The $X$ coordinate and the $Y$ coordinate are calculated based on the similarity relationship of triangle.

$$
\begin{aligned}
& X=\frac{L}{d} x \\
& Y=\frac{L}{d} y \\
& Z=L+d
\end{aligned}
$$

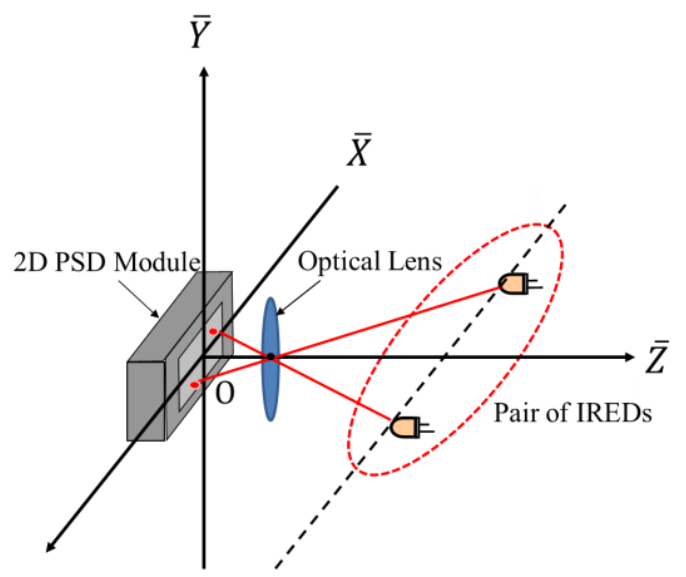

(a) Schematic view

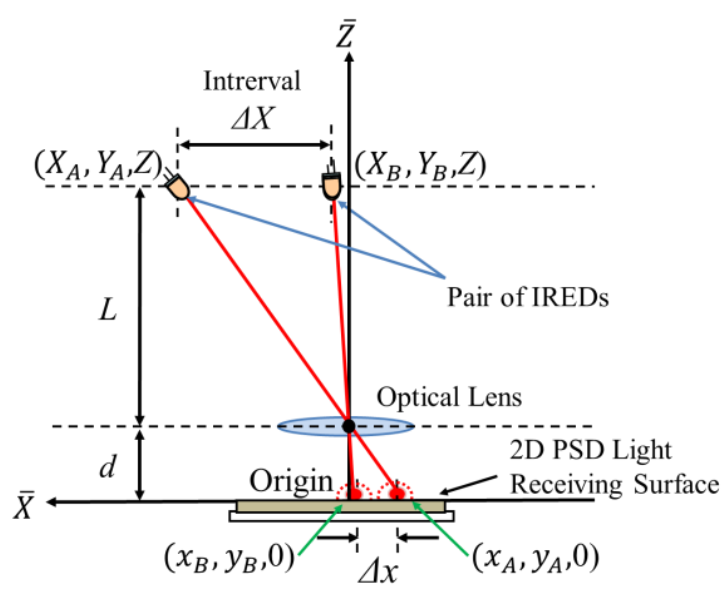

(b) X-Z plane

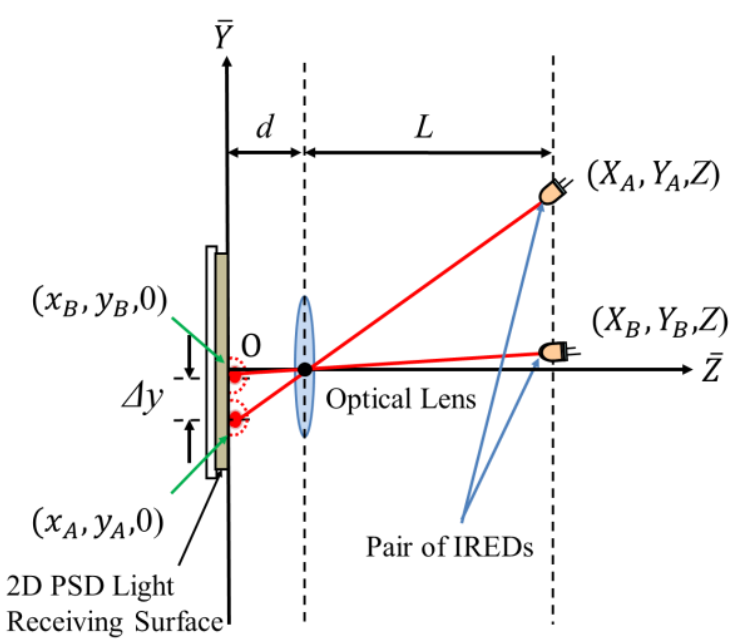

(c) Y-Z plane

Fig. 1. Positional relationship between light targets and PSD.

However, there is the condition for the use of this principle. A pair of light sources must be parallel to the 2D PSD light reception surface of the 2D PSD. 


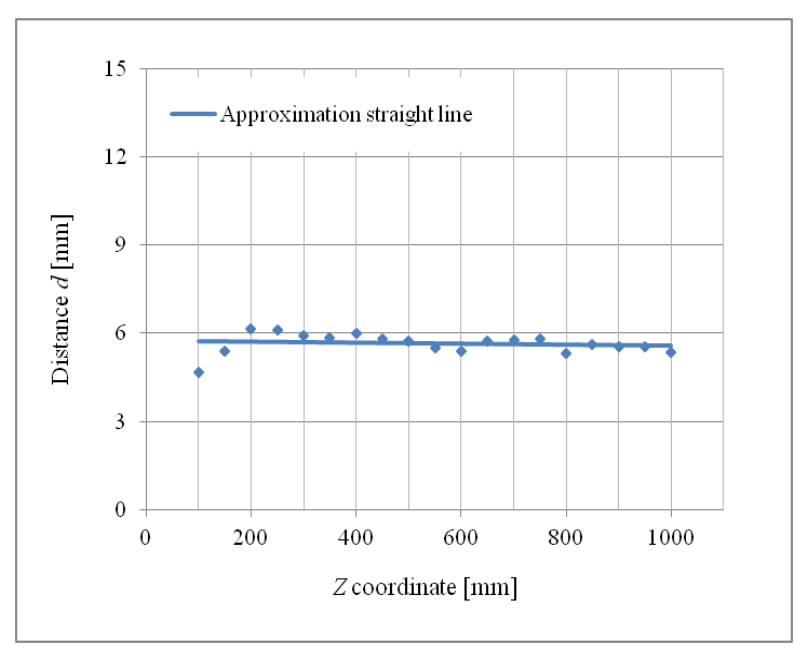

Fig. 2. Measurement result of the distance $d$.

\subsection{Preset of the distance $d$}

The spot light 2D coordinate $(x, y)$ on the 2D PSD light reception surface, the interval $\Delta x$, the distance $L$, the 3D coordinate of the light source $(X, Y, Z)$ are successively sequentially obtained during the system operation. However, the distance $\mathrm{d}$ is required to be preset. The $Z$ coordinate of the light source was measured by using the ruler. From the equation (4) and (3), the distance $d$ is obtained.

$$
d=\left(1+\frac{\Delta x}{\Delta X}\right)^{-1} Z
$$

In this system, we was set to the light source interval $\Delta X=$ $5[\mathrm{~cm}]$. We fixed the $X$ coordinate of a pair of light source to each $-2.5[\mathrm{~cm}]$ and $2.5[\mathrm{~cm}]$, the $Y$ coordinate to $0[\mathrm{~cm}]$. And we changed the $Z$ coordinate from $10[\mathrm{~cm}]$ to $100[\mathrm{~cm}]$ in the step size of $5[\mathrm{~cm}]$. We show the distance $d$ measurement result in Fig. 2. From Fig. 2, we found that the distance $d$ was the certain value. Therefore, the distance $d$ was $5.70[\mathrm{~mm}]$.

\section{Measuring system}

The measurement system is configured the light projecting unit, the light receiving unit and the calculating unit. We show the measurement system configuration diagram in Fig. 3.

\subsection{Light projecting unit}

The frequency modulation circuit can modulate the frequency of the light source into an optional frequency. And, we project the light that is frequency modulated toward the light receiving unit.
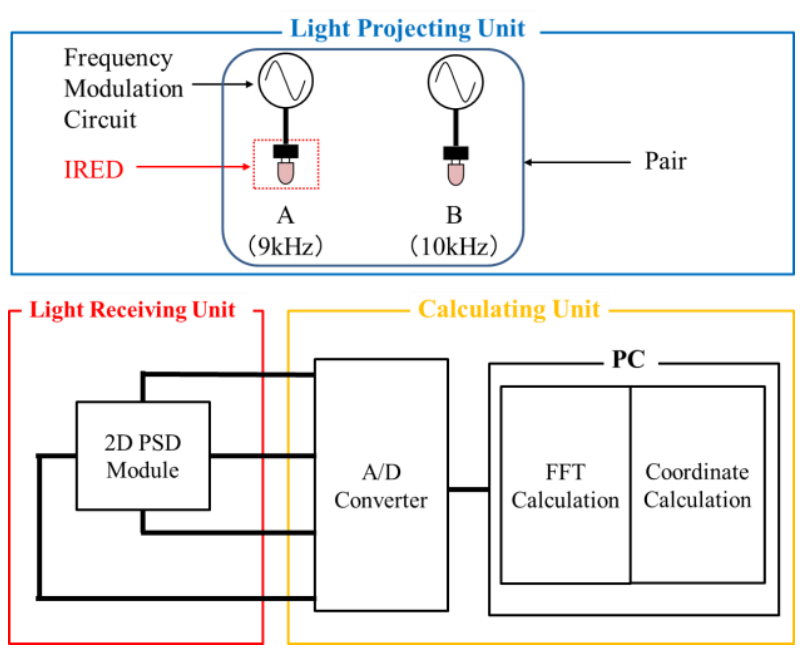

Fig. 3. Measuring system configuration diagram

\subsection{Light receiving unit}

The projected light passes through the optical lens, and is imaged on the 2D PSD light receiving surface of the light receiving unit. Then, electric current corresponding to the spotlight coordinate on the 2D PSD module light-receiving surface is outputted from the 2D PSD. The electric current is converted voltage in the $2 \mathrm{D}$ module. Four signals output from the 2D PSD module are sent to the A/D converter of the calculating unit to detect the $2 \mathrm{D}$ coordinate of the spot light on the 2D PSD light reception surface.

\subsection{Calculating unit}

The amplitude data converted by the A/D converter is stored in the memory of the A/D converter. Then, 1024 amplitude data is taken from each of the output signal of the 2D PSD module, these data are sent to the PC after 4096 amplitude data are stored in memory.

Amplitude data is FFT calculation on the PC. Then, 4 spectrums are obtained at the modulation frequency of each light source from the composite wave. 3D coordinate $(X, Y$, $Z$ ) of each light source is determined by using their spectral peak voltages.

\section{Experiments}

We verified accuracy of the $3 \mathrm{D}$ coordinate detection. Theoretical value of the light source coordinate is the value that was measured using graph paper and the ruler. We were measured under fluorescent lamps. A pair of light sources was used the light source modulated to $10[\mathrm{kHz}]$ and $9[\mathrm{kHz}]$. Because the $Y$ coordinate are detected by the same principle 
as principle of the $X$ coordinate, we omit the $Y$ coordinate measurement.

\subsection{Measurement accuracy verification}

We verified accuracy of the $3 \mathrm{D}$ coordinate detection using infrared-emitting diodes (IREDs) which were 22 $[\mathrm{mW} / \mathrm{Sr}]$. Also, a pair of light sources interval $\Delta X$ was set 5 $[\mathrm{cm}]$.

First, we verified measurement accuracy of the $Z$ coordinate of the light sources. The $X$ coordinate of a pair of IREDs were set $-2.5[\mathrm{~cm}]$ and $+2.5[\mathrm{~cm}]$, The $Y$ coordinate was set $0[\mathrm{~cm}]$. Moreover, the $Z$ coordinate was changed from $10[\mathrm{~cm}]$ to $100[\mathrm{~cm}]$ at an interval of $5[\mathrm{~cm}]$. We show the $Z$ coordinate measurement result in Fig. 4.

Second, we verified measurement accuracy of the $X$ coordinate of the light sources. The $Y$ coordinate of a pair of IREDs were set $0[\mathrm{~cm}]$, the $Z$ coordinate was set $20[\mathrm{~cm}]$. Moreover, the $X$ coordinate changed from $-20[\mathrm{~cm}]$ to +20 [cm] at an interval of $5[\mathrm{~cm}]$. In the similar way, the $X$ coordinate was measured when the $Z$ coordinate was 50 [cm] and $80[\mathrm{~cm}]$. We show the $X$ coordinate measurement result when the $Z$ coordinate was $20[\mathrm{~cm}], 50[\mathrm{~cm}], 80[\mathrm{~cm}]$ in Fig. 5(a), (b), (c).

First, we describe the $Z$ coordinate measurement result. The maximum error of the $Z$-coordinate measurement results was 133 [mm], the maximum error rate was 17 [\%].

Second, we describe the $X$ coordinate measurement result when the $Z$ coordinate was $20[\mathrm{~cm}]$. 3D coordinate could not be measured because a pair of light sources were not entered into the view angle of the camera when the $X$ coordinate was $15[\mathrm{~cm}]$ or more in absolute value.

Furthermore, the maximum measurement error of the maximum measurement error rate was 17.7 [\%] within the

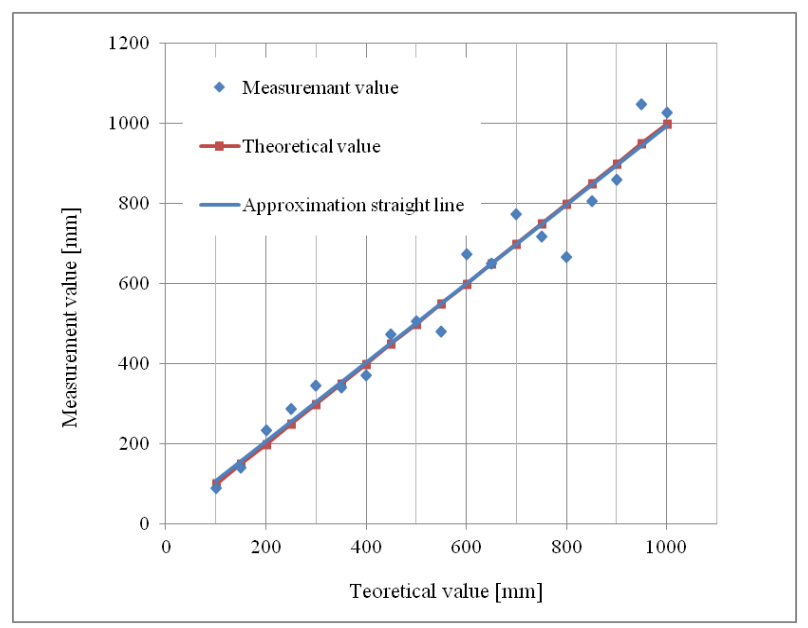

Fig. 4. Measurement result of the $Z$-coordinate.

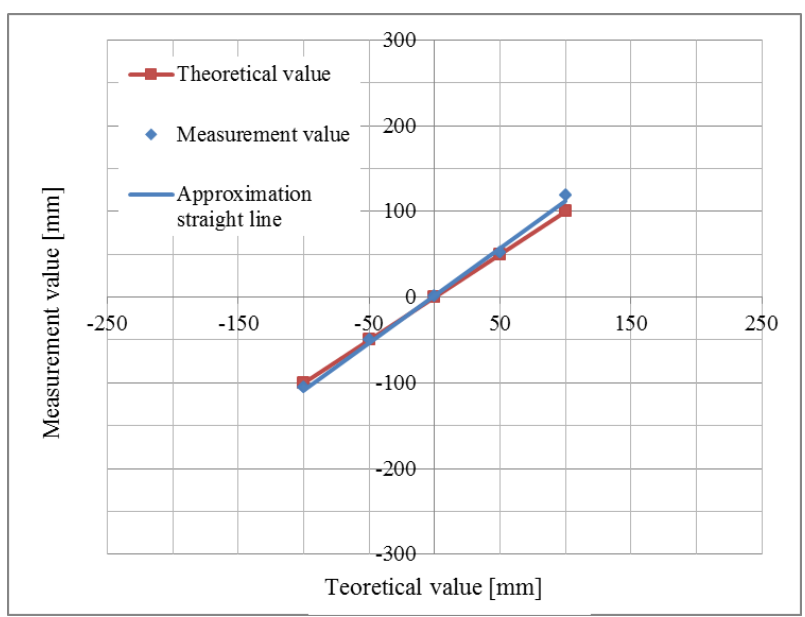

(a) $Z=20[\mathrm{~cm}]$

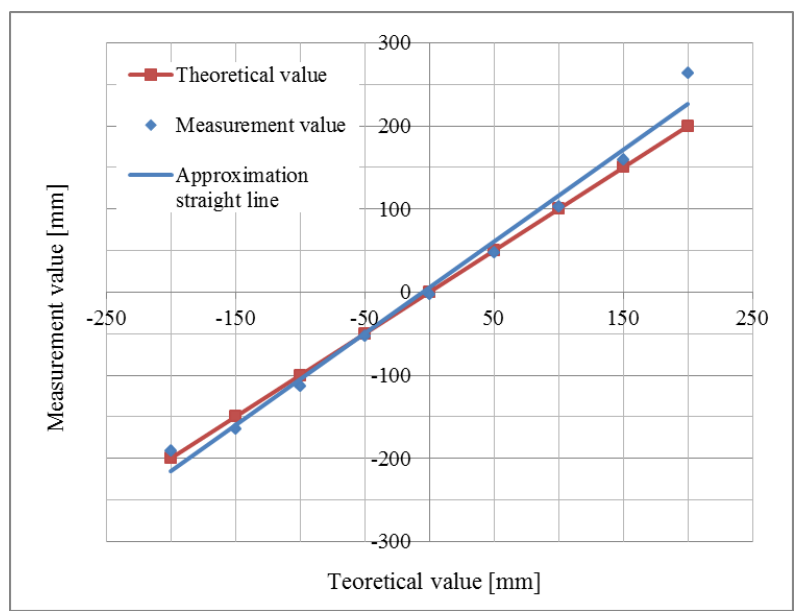

(b) $Z=50[\mathrm{~cm}]$

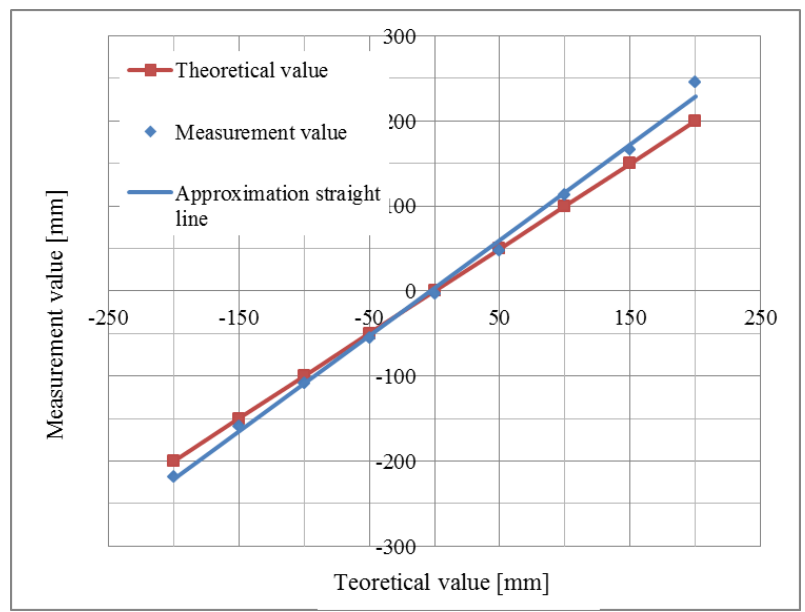

(c) $Z=80[\mathrm{~cm}]$

Fig. 5. Measurement result of the $X$-coordinate.

measuring range.

Third, when the $Z$ coordinate was $50[\mathrm{~cm}]$, the maximum measurement error of the $X$-coordinate measurement results was $63.3[\mathrm{~mm}]$, the maximum 
measurement error rate was 31.7 [\%].

Finally, when the $Z$ coordinate was $80[\mathrm{~cm}]$, the maximum measurement error of the $X$-coordinate measurement results was $45.9[\mathrm{~mm}]$, the maximum measurement error rate was 23.0 [\%].

From the above, the measurement error markedly appeared as the light sources separated farther from the 2D PSD light reception surface. Therefore, the measurement error cause was correlation with the incident light quantity. Furthermore, another measurement error cause is that the spot light size was larger than 2D PSD light reception surface. The graph of the average value almost coincided with the approximation graph of the theoretical value. Therefore, the proposed method is an effective 3D measurement method.

\subsection{The measurement accuracy verification on a pair of the light source interval $\Delta X$}

We compared the 3D position detection accuracy when a pair of light sources interval $\Delta X$ was $5[\mathrm{~cm}], 10[\mathrm{~cm}], 20$ [cm]. a pair of the light sources were used IREDs which were $22[\mathrm{~mW} / \mathrm{Sr}]$.

First, we measured the $Z$ coordinate of the IREDs when the light source interval $\Delta X$ was 10 [cm]. The $X$ coordinate of a pair of IREDs were set $-5[\mathrm{~cm}]$ and $+5[\mathrm{~cm}]$, The $Y$ coordinate was set $0[\mathrm{~cm}]$. Moreover, the $Z$ coordinate was changed from $10[\mathrm{~cm}]$ to $100[\mathrm{~cm}]$ at an interval of $5[\mathrm{~cm}]$.

Second, we measured the $Z$ coordinate of the IREDs when the light source interval $\Delta X$ was $10[\mathrm{~cm}]$. The $X$ coordinate of a pair of IREDs were set $-10[\mathrm{~cm}]$ and +10 [cm], The $Y$ coordinate was set $0[\mathrm{~cm}]$. Moreover, the $Z$ coordinate was changed from $10[\mathrm{~cm}]$ to $100[\mathrm{~cm}]$ at an interval of $5[\mathrm{~cm}]$.

We show the $Z$ coordinate measurement result when a pair of light sources interval $\Delta X$ was $10[\mathrm{~cm}], 20[\mathrm{~cm}]$ in Fig. 6(a), (b).

The 3D coordinate could not be measured because a pair of light sources was not entered into the view angle of the camera when the $Z$ coordinate was 10 [cm] and 15 [cm]. From Fig. 5 and Fig. 6, the wider the light source interval $\Delta X$ becomes, the more the measurement accuracy was improved. The spot light interval on the 2D PSD light reception surface was widened by widening the light source interval $\Delta X$. Therefore, because the influence of the spot light displacement or quantization error was suppressed, the measurement accuracy was improved. However, if the light source interval is set excessively wide, it is difficult to enter a pair of the spot light on the 2D PSD light reception

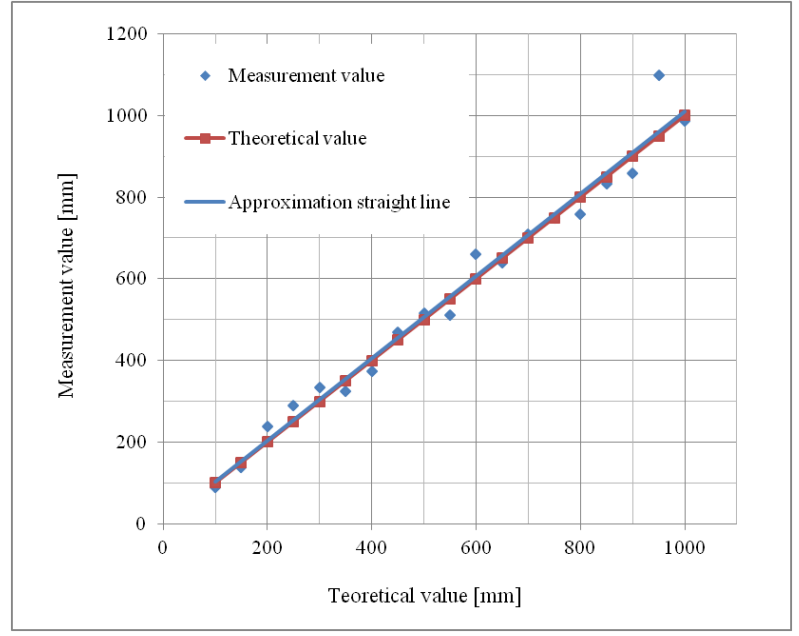

(a) $\Delta X=10[\mathrm{~cm}]$

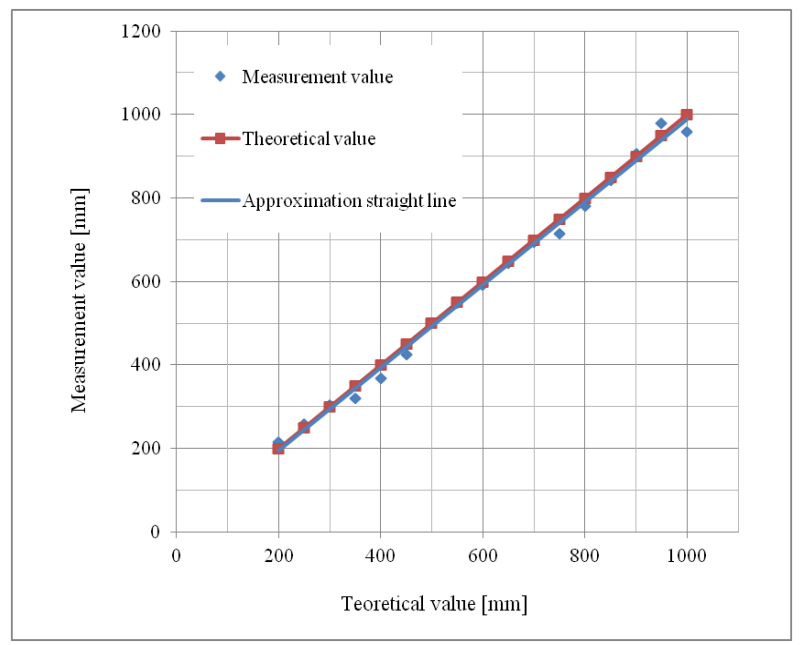

(b) $\quad \Delta X=20[\mathrm{~cm}]$

Fig. 6. Accuracy comparison on a pair of the light source interval $\Delta X$

surface. As the result, 3D measurement range is narrowed. Therefore, the light source interval $\Delta X$ must be set to an appropriate value corresponding to application or the purpose.

\subsection{The measurement accuracy verification on luminance of a pair of the light sources}

We compared the 3D position detection accuracy in the case of using low luminance IREDs which were 22 [mW/Sr] to the 3D position detection accuracy in the case of high luminance IREDs which were 40 [mW/Sr].

First, the $Z$ coordinate light sources were measured. The $X$ coordinate of a pair of light sources were set -2.5 [cm] and $+2.5[\mathrm{~cm}]$, The $Y$ coordinate of the light sources were set $0[\mathrm{~cm}]$. Moreover the $Z$ coordinate of the light 
sources were changed from $10[\mathrm{~cm}]$ to $100[\mathrm{~cm}]$ at an interval of $5[\mathrm{~cm}]$. We show the $Z$ coordinate measurement result in Fig. 7.

Second, we measured the $X$ coordinate light sources. The $Y$ coordinate of the light sources were set $0[\mathrm{~cm}]$, The $Z$ coordinate of the light sources were set $20[\mathrm{~cm}]$. Moreover the $X$ coordinate of the light sources changed from $-20[\mathrm{~cm}]$ to $+20[\mathrm{~cm}]$ at an interval of $5[\mathrm{~cm}]$. In the similar way, the $X$ coordinate was measured when the $Z$ coordinate was 50 $[\mathrm{cm}]$ and $80[\mathrm{~cm}]$. We show the $X$ coordinate measurement result when the $Z$ coordinate was $20[\mathrm{~cm}], 50[\mathrm{~cm}], 80[\mathrm{~cm}]$ in Fig. 8(a), (b), (c).

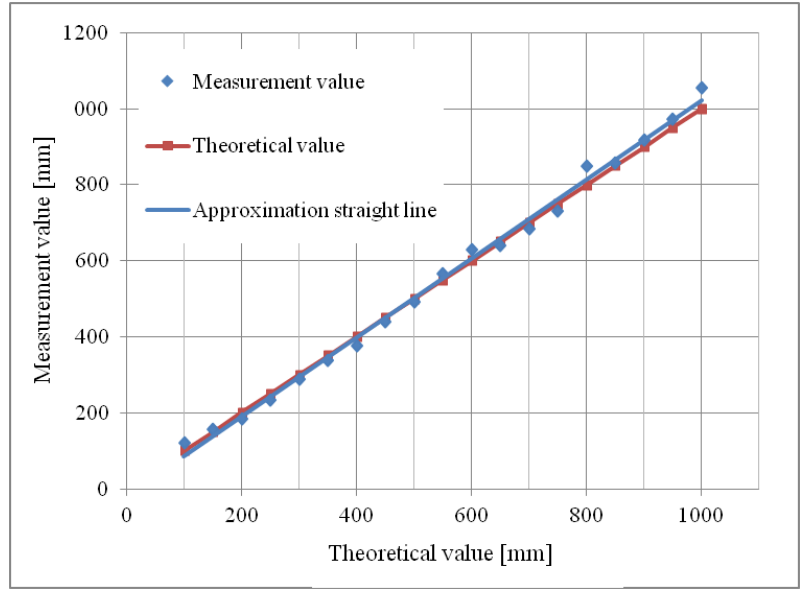

Fig. 7. Measurement result of the Z-coordinate.

First, we describe the $Z$ coordinate measurement result. From Fig. 7, the maximum measurement error was 54.1 [mm] and the maximum measurement error rate was 6.8 [\%] in the case of using the high luminance IREDs. Fig. 4 and Fig. 7, the measurement error was improved $78.9[\mathrm{~mm}]$, the measurement error rate was improved 10.2 points in the case of using the high luminance IREDs.

Second, we describe the $X$ coordinate measurement result when the $Z$ coordinate was $20[\mathrm{~cm}]$. 3D coordinate could not be measured because a pair of light sources were not entered into the view angle of the camera when the $X$ coordinate was $15[\mathrm{~cm}]$ or more in absolute value. From Fig. 8 (a), the maximum measurement error was 10.7 [mm] and the maximum measurement error rate was 10.7 [\%] in the case of using the high luminance IREDs. Fig. 5(a) and Fig. 8(a), the measurement error was improved 3.0 [mm], the measurement error rate was improved 3.0 points in the case of using the high luminance IREDs.

Third, we describe the $X$ coordinate measurement result when the $Z$ coordinate was 50 [cm]. From Fig. 8(b), the maximum measurement error was 10.1 [mm] and the

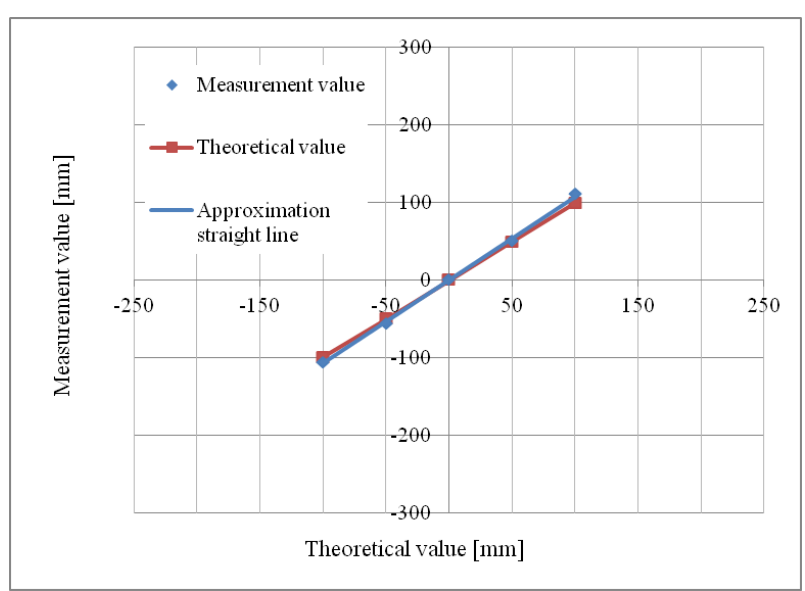

(a) $Z=20[\mathrm{~cm}]$

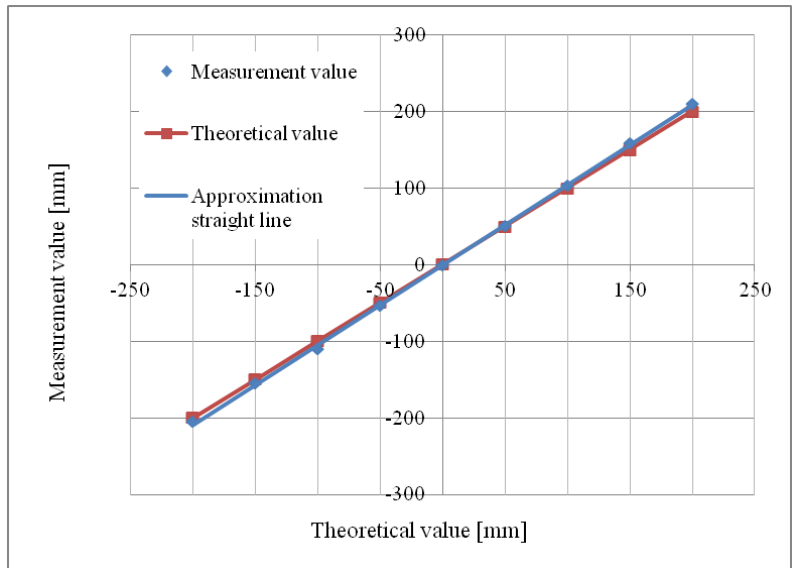

(b) $Z=50[\mathrm{~cm}]$

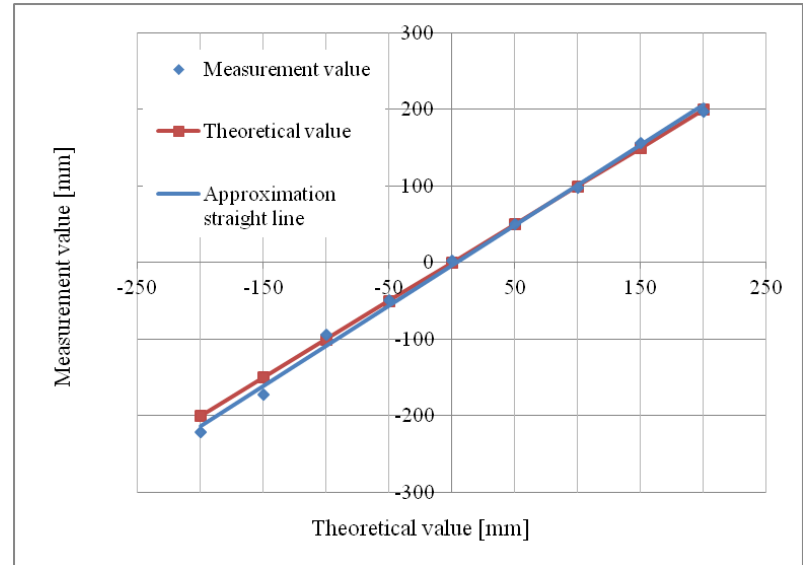

(c) $Z=80[\mathrm{~cm}]$

Fig. 8. Measurement result of the $X$-coordinate

maximum measurement error rate was 9.5 [\%] in the case of using the high luminance IREDs. Fig. 5(b) and Fig. 8(b), the measurement error was improved $53.2[\mathrm{~mm}]$, the 
measurement error rate was improved 22.2 points in the case of using the high luminance IREDs.

Finally, we describe the $X$ coordinate measurement result when the $Z$ coordinate was 80 [cm]. From Fig. 8(c), the maximum measurement error was $21.6[\mathrm{~mm}]$ and the maximum measurement error rate was 10.8 [\%] in the case of using the high luminance IREDs. Fig. 5(c) and Fig. 8(c), the measurement error was improved 24.3 [mm], the measurement error rate was improved 12.2 points in the case of using the high luminance IREDs.

In every case, the measurement accuracy was superior in the case of using the higher luminance IREDs. From the above, it was confirmed that the measurement accuracy was correlation with the incident light quantity. The position resolution of the $2 \mathrm{D}$ PSD is changed by the signal photocurrent value. Furthermore, the signal photocurrent value and the incident light quantity are in proportion. Therefore, the measurement accuracy is correlation with the incident light quantity.

\section{Conclusions}

In this study, we proposed the new 3D measurement method with a 2D PSD using a pair of light sources. Therefore, the 3D measurement was achieved at simple preset, high speed and low cost. However, this proposed method is the conditional method that a pair of light sources must be arranged in parallel to the 2D PSD light reception surface. In the case that a pair of light sources is not arranged in parallel to the 2D PSD light reception surface, it is our future work to develop the measurement system capable of more accurately the 3D measurement. Furthermore, it is also our future work to develop a measurement system capable of real-time 3D measurement in a wider range.

\section{References}

(1) Yuya Kawahara, and Ryuichi Udo, and Lifeng Zhang : "High speed 3D Light Source Position Detection by Combining a 2D PSD an Ultrasonic Sensor Unit", Journal of the Institute of Industrial Applications Engineers, Vol. 2, No. 1, pp. 42-47, 2014

(2) Hua Li, and Jian Zhang, and Da Xu, and Mengchun Zhong : "Study on Laser Spot Affecting the Accuracy of Transient Angle Test System Based on PSD", 3rd International Conference on Electric and Electronics (EEIC 2013), pp. 477-479, 2013
(3) Jeong-Gi Lee, and Do-Myung Kim, and Jin-Young Suk : "Development of a 3D Position Determination Device using PSD sensor", Control Automation and Systems (ICCAS), 2010 International Conference on, pp. 1733-1736, 2010

(4) Ying-gang Zhou, and Yan-gen Xie, and Dan Zhan, and Guang-ping Yu : "Research of 3D Spatial Localizing System Based on PSD Sensor”, Measuring Technology and Mechatronics Automation, 2009. ICMTMA '09. International Conference on, Vol. 1, pp. 55-58, 2009

(5) Jong Kyu Jung, and Seong gu Kang, and Joon Sik Nam, and Kyi Hwan Park : "Intensity Control of Triangulation Based PSD Sensor Independent of Object Color Variation", Sensors Journal, IEEE, Vol. 11, No. 12, pp. 3311-3315, 2011 